Claremont Colleges

Scholarship@ Claremont

All HMC Faculty Publications and Research

HMC Faculty Scholarship

5-1-2005

\title{
X-Ray Generation from Metal Targets Coated with Wavelength-Scale Spheres
}

D. R. Symes

University of Texas at Austin

H. A. Sumeruk

University of Texas at Austin

I. V. Churina

University of Texas at Austin

Thomas D. Donnelly

Harvey Mudd College

J. Landry

University of Texas at Austin

See next page for additional authors

\section{Recommended Citation}

D. R. Symes, H.A. Sumeruk, I.V. Churina, A. Belolipetski, Stefan Kneip, J. Landry, T.D. Donnelly, T. Ditmire, "X-Ray Generation from Metal Targets Coated with Wavelength-Scale Spheres," CLEO/QELS (Baltimore, Maryland, 2005). doi: 10.1109/

QELS.2005.1549203

This Conference Proceeding is brought to you for free and open access by the HMC Faculty Scholarship at Scholarship @ Claremont. It has been accepted for inclusion in All HMC Faculty Publications and Research by an authorized administrator of Scholarship @ Claremont. For more information, please contact scholarship@cuc.claremont.edu. 
Authors

D. R. Symes, H. A. Sumeruk, I. V. Churina, Thomas D. Donnelly, J. Landry, and T. Ditmire 


\title{
X-Ray Generation from Metal Targets Coated with Wavelength-Scale Spheres
}

\author{
D. R. Symes, H. A. Sumeruk, I. V. Churina, T. D. Donnelly, J. Landry and T. Ditmire \\ Fusion Research Center, Department of Physics, University of Texas at Austin, 1 University Station C1510, Austin, Texas, 78712, U.S.A. \\ dansymes@mail.utexas.edu
}

\begin{abstract}
X-ray yield measurements from targets coated with wavelength-scale spheres are compared with measurements from polished targets. Evidence for a hotter resonant electron temperature due to field enhancements from Mie resonances in the spheres is investigated. (C) 2004 Optical Society of America

OCIS codes: (320.7120) Ultrafast phenomena; (350.5400) Plasmas
\end{abstract}

\section{Introduction}

A major application of plasmas produced by high-intensity laser pulses is the generation of short bursts of $\mathrm{x}$-rays. For efficient hard $\mathrm{x}$-ray $(>\mathrm{few} \mathrm{keV})$ production the acceleration of hot electrons from resonant absorption processes should be optimized. Recent experiments have demonstrated enhancements in x-ray yield achieved by using structured surfaces such as gratings [1], velvets [2], and nanoparticles [3].

In this paper, we investigate hard $\mathrm{x}$-ray $(>100 \mathrm{keV})$ generation from copper targets coated with a partially covering layer of microspheres. When a spherical object is illuminated with light of a wavelength, $\lambda$, similar to the diameter, $d$, of the object, a complicated electric field pattern arises due to interference between the incident and scattered waves. The interaction of an intense laser with such a target can generate a spherical plasma with nonuniform heating across its surface $[4,5]$. For example, Fig. 1 displays the electric field pattern of a $1 \mu \mathrm{m}$ spherical plasma irradiated with $0.8 \mu \mathrm{m}$ laser light. In particular, when the size parameter $m=\pi d / \lambda \approx 1$, the system can experience a Mie resonance in which the electric fields are greatly enhanced. By depositing wavelength-length scale spheres across the surface of a metal substrate, we aim to exploit the Mie resonance to increase the temperature of the resonantly heated electrons which are responsible for the generation of hard $\mathrm{x}$-rays.
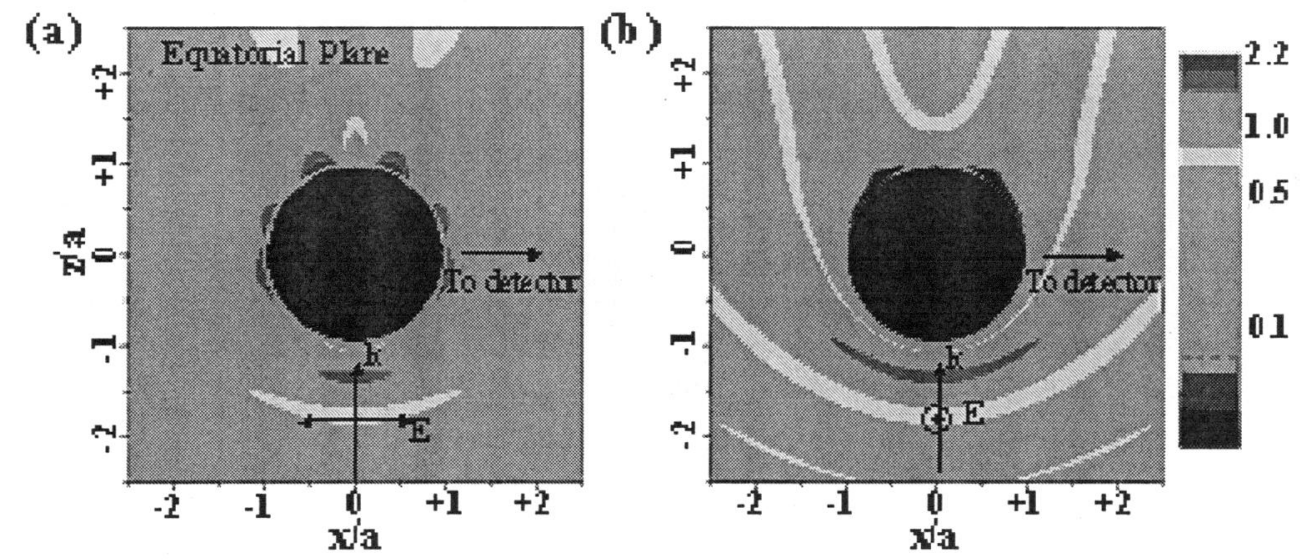

Fig. 1. Calculated electric field plot for an ionized, micron-diameter plasma sphere for laser polarization (a) parallel and (b) perpendicular to the page. The laser propagation direction is $+z$. Note the enhancement in the field visible in (a).

\section{Target preparation}

We prepared the targets using commercially available polystyrene spheres of diameter $0.26,0.5,1.0,1.5$ and $2.9 \mu \mathrm{m}$ with a size distribution of $3 \%$, diluted with water to a concentration of $\approx 1: 1000$, and applied to polished copper substrates. The solution was made more dilute for the smaller spheres to improve the consistency of the surface. We observed the target surfaces using a scanning electron microscope (SEM) to ascertain the uniformity of the coating and to check the depth of the sphere layers. SEM images of copper coated with a $2.9 \mu \mathrm{m}$ sphere solution, shown in Fig. 2, at magnification of (a) 100x and (b) 5000x demonstrate that the spheres form a monolayer over a large area. 
(a)

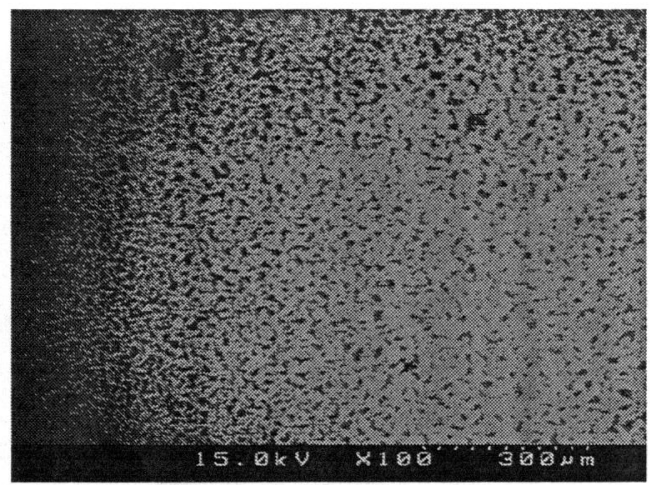

(b)

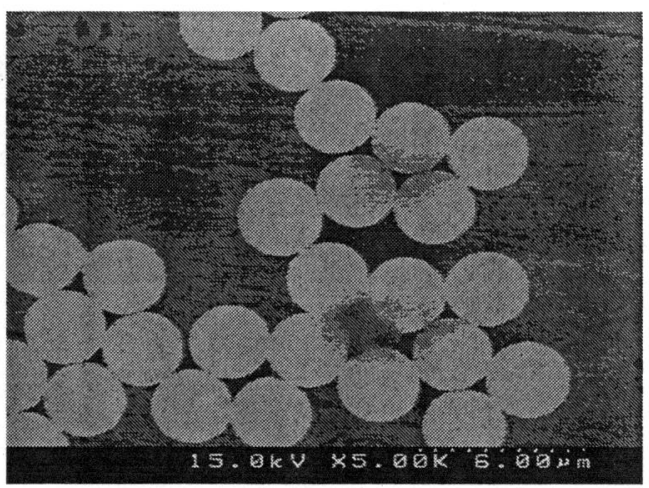

Fig. 2. Scanning electron microscope images of a copper substrate coated with $2.9 \mu \mathrm{m}$ spheres at (a) $100 \mathrm{x}$ and (b) $5000 \mathrm{x}$ magnification. Note the uniformity of coverage in (a) and the size consistency shown in (a).

\section{Experiment}

We performed the experiment using the THOR laser system at the University of Texas which provided 35 fs pulses at a wavelength of $800 \mathrm{~nm}$. The pre-compression energy was set at $\approx 68 \mathrm{~mJ}$ yielding a focal intensity $\approx 1 \times 10^{18} \mathrm{Wcm}^{-2}$. This limit was required to avoid saturation of the $\mathrm{x}$-ray detectors.

$\mathrm{X}$-ray emission from the plasma was observed using six filtered $\mathrm{NaI}$ scintillation detectors arranged around the target chamber. The filters were composed of $125 \mathrm{~mm} \mathrm{Al}(450 \mathrm{keV}), 12 \mathrm{~mm} \mathrm{Cu}(162 \mathrm{keV}), 32 \mathrm{~mm} \mathrm{Cu}(500 \mathrm{keV})$, $43 \mathrm{~mm} \mathrm{Cu}(600 \mathrm{keV}), 24 \mathrm{~mm} \mathrm{~Pb}(700 \mathrm{keV})$ and $44 \mathrm{~mm} \mathrm{~Pb}(900 \mathrm{keV})$, where the bracketed values are the $1 / \mathrm{e}^{2}$ cutoff energies of each filter after accounting for any extra material between the detector and the target.

Ion emission from the front surface of the target was measured using a charge collector (Faraday cup) at a distance of $0.20 \mathrm{~m}$ behind a mesh biased at $-300 \mathrm{~V}$ to reduce the emission of secondary electrons from the detector surface. A magnetic field of $1 \mathrm{kG}$ was placed $\approx 1 \mathrm{~cm}$ in front of the collector to deflect electrons.

\section{Results}

We irradiated polished copper targets at laser incidence angles between $0^{\circ}$ and $45^{\circ}$ from the target normal to ascertain the optimum angle for hard $\mathrm{x}$-ray production under our conditions. The maximum $\mathrm{x}$-ray emission occurred for an incident angles $\approx 10^{\circ}-20^{\circ}$ suggesting that the dominant mechanism for hot electron generation was resonance absorption. The signal levels reduced towards $45^{\circ}$ indicating that vacuum heating was inefficient. This was probably due to the formation of a preplasma caused by the interaction of a low level prepulse.

$\mathrm{X}$-ray yields and ion spectra from polished copper targets were then directly compared to sphere coated copper targets at incidence angles of $0^{\circ}, 10^{\circ}, 24^{\circ}$ and $45^{\circ}$. Initial results using $2.9 \mu \mathrm{m}$ spheres are shown in Figs. 3 and 4. Fig. 3 shows the $\mathrm{x}$-ray yield through the $32 \mathrm{~mm} \mathrm{Cu}$ filter corresponding to an integrated signal of photon energies above $\approx 500 \mathrm{keV}$. The sphere-coated targets display a clear enhancement in yield at all four angles of incidence of the laser beam, even where resonance absorption is expected to be optimized in the polished copper target. This suggests that the presence of the spheres not only modifies the target surface allowing resonant processes at off-resonant angles but also increases the magnitude of the driving fields through an interference effect (Mie resonance) as predicted. We note that the size parameter for these spheres is relatively large, $m=11$, so the effect for smaller spheres should be even more significant. 


\section{JThD3}

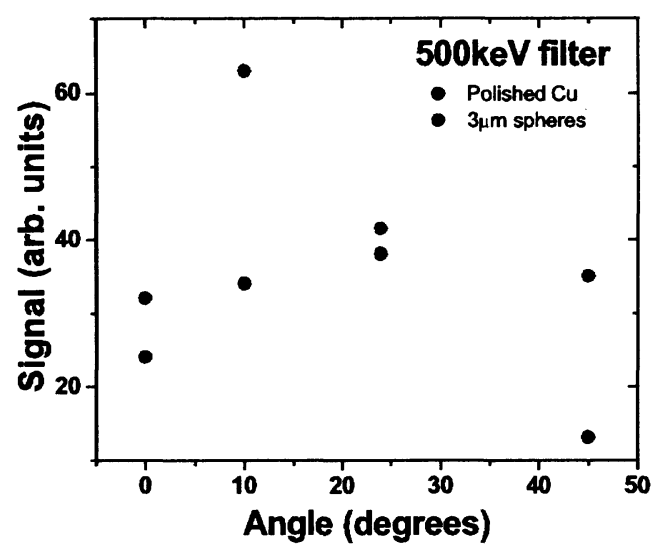

Fig. 3. Hard $\mathrm{x}$-ray yields versus angle of incidence of the laser beam for polished copper targets and $2.9 \mu \mathrm{m}$ sphere coated copper targets.

Time-of-flight (TOF) traces from polished copper and $2.9 \mu \mathrm{m}$ sphere-coated copper, shown in Fig. 4, for an incident angle of $45^{\circ}$ differed considerably. The earliest time we could resolve after the photon and hot electron burst was $\approx 1.12 \mu \mathrm{s}$. This TOF corresponds to protons of energy $\approx 200 \mathrm{eV}$, below which protons would be deflected by the $1 \mathrm{kG}$ magnetic field. The maximum visible energy of $\approx 10 \mathrm{keV}$ (assuming the ion mass of copper) is too low considering the much higher electron energies implied by the $x$-ray measurements. This suggests that we only see the colder ions from a distribution driven by two electron temperatures [6]. It is likely that the TOF trace from the sphere targets is predominantly carbon and oxygen whereas the signal from the metal may contain all three species (since contaminants will be present [7]). However, when we irradiated copper coated with a thin plastic film $(13 \mu \mathrm{m})$, no ion signal was visible implying that the entire trace shown is due to copper ions. This would imply a significant shift in the thermal temperature of the plasma between the two cases and an energy spectrum calculated assuming that all the signal comes from copper ions is shown in the inset.

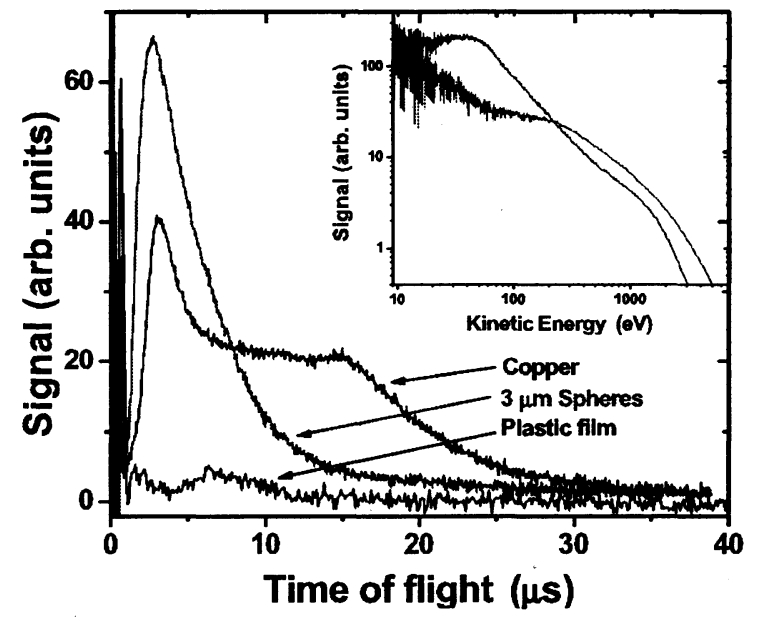

Fig. 4. Time-of-flight traces for polished copper targets and $2.9 \mu \mathrm{m}$ sphere coated copper targets. (Inset) Kinetic energy spectra assuming Cu ions.

[1] S. P. Gordan, T. Donnelly, A. Sullivan, H. Hamster, and R. W. Falcone, "X rays from microstructured targets heated by femtosecond lasers," Op. Lett. 19, 484 (1994).

[2] G. Kulcsar, D. AlMawlawi, F. W. Budnik, P. R. Herman, M. Moskovits, L. Zhao, and R. S. Marjoribanks, "Intense Picosecond X-Ray Pulses from Laser Plasmas by Use of Nanostructured "Velvet" Targets," Phys. Rev. Lett. 84, 5149 (2000).

[3] P. P. Rajeev, P. Taneja, P. Ayyub, A. S. Sandhu, and G. Ravindra Kumar, "Metal Nanoplasmas as Bright Sources of Hard X-Ray Pulses," Phys. Rev. Lett. 90, 115002 (2003).

[4] T. D. Donnelly, M. Rust, I Weiner, M. Allen, R. A. Smith, C. A. Steinke, S. Wilks, J. Zweiback, T. E. Cowan, and T. Ditmire, "Hard X-Ray and Hot Electron Production from Intense Laser Irradiation of Wavelength-Scale Particles," J. Phys. B 34, L313 (2001).

[5] D. R. Symes, A. J. Comley, and R. A. Smith, "Fast-Ion Production from Short-Pulse Irradiation of Ethanol Microdroplets," Phys. Rev. Lett. 93, 145004 (2004).

[6] A. A. Andreev and J. Limpouch, "Ion Acceleration in Short-Pulse Laser-Target Interactions," J. Plasma Physics 62, 179 (1999).

[7] E. L. Clark, K. Krushelnick, M. Zepf, F. N. Beg, M. Tatarakis, A. Machacek, M. I. K. Santala. I. Watts. P. A. Norreys, and A. E. Dangor, "Energetic Heavy-Ion and Proton Generation from Ultraintense Laser-Plasma Interactions with Solids," Phys. Rev. Lett. 85, 1654 (2000). 